Check for updates

Cite this: RSC Adv., 2017, 7, 28889

\title{
Excellent rheological performance and impact toughness of cellulose nanofibers/PLA/ionomer composite
}

\author{
Jingjing $L i, \uparrow^{a}$ Jian $L i, \uparrow^{a}$ Dejun Feng, ${ }^{a}$ Jingfeng Zhao, ${ }^{a}$ Jingrong Sun ${ }^{a}$ \\ and Dagang $\mathrm{Li}(\mathbb{D} * \mathrm{~b}$
}

Cellulose nanofibers (CNFs) with high aspect ratio were used to reinforce polylactic acid (PLA) by extrusion. Surlyn ionomer was adopted to improve the interfacial adhesion and impact toughness of the CNFs/PLA composite. To overcome the aggregation of CNFs, a water preblending process was carried out before the extrusion. The rheological results showed the ionomer can decrease the viscosity of the CNFs/PLA composite and improve the flowability due to the lubrication of the ionomer. The bending strength (MOR) and bending modulus (MOE) of the composite with ionomer were much higher than those of a composite with maleic anhydride grafted polyethylene (MAPE). In addition, the CNFs/PLA/ionomer composite showed extremely high impact toughness, which was up to $34.2 \mathrm{~J} \mathrm{~m}^{-1}$ when the loading of CNFs was 30 wt\%, increasing by $302.3 \%$ as compared to neat PLA. FE-SEM images demonstrated the homogenous dispersion of CNFs in PLA by using the water preblending method.

Received 17th April 2017

Accepted 25th May 2017

DOI: $10.1039 / \mathrm{c} 7 \mathrm{ra0} 4302 \mathrm{c}$

rsc.li/rsc-advances

(such as, plasma) and chemical modification (such as, esterifi-

\section{Introduction}

Nowadays, the production of sustainable and environmentally friendly materials has generated enormous attention in both academic and industrial fields. As a typical eco-friendly material, polylactic acid (PLA) has been rapidly developed for the past few decades. PLA is a biodegradable, thermoplastic, and aliphatic polyester, and it can be derived from renewable resources, such as starch. ${ }^{1}$ PLA has already been found in many applications, including textile, medical, and packaging fields. ${ }^{2}$ Nonetheless, the inherent brittleness of PLA has been the main obstacle to expand its commercial use.

Cellulose nanofibers (CNFs) derived from renewable biomass have attracted much interest as an alternative in composite materials. Numerous studies have focused on the use of CNFs as reinforcement in PLA, and the nanocomposites have been produced by melt compounding and solvent casting. ${ }^{1-7}$ Although CNFs have a great potential as reinforcement, the uniform dispersion of CNFs in polymer matrix is still the major challenge. CNFs can not be uniformly dispersed in thermal plastic polymers due to high polarity of cellulose surface and the resultant low interfacial compatibility with hydrophobic polymer matrix. ${ }^{8}$ To overcome the compatibility difficulty, cellulose has been subjected to physical treatment

${ }^{a}$ College of Forestry, Northwest A\&F University, Yangling 712100, Shaanxi, China ${ }^{b}$ College of Materials Science and Engineering, Nanjing Forestry University, Nanjing 210037, Jiangsu, China.E-mail: 396420748@qq.com

$\dagger$ Jingjing $\mathrm{Li}$ and Jian $\mathrm{Li}$ contributed equally to this work and should be considered co-first-authors. cation, etherification, oxidation, silylation, and polymer grafting by click chemistry). ${ }^{9}$ However, the mechanical properties of the prepared nanocomposites are not significantly improved due to the damaged molecular structure of CNFs by chemical modification. In addition, chemical modification is not an ecofriendly way to prepare the all-green composite.

In this study, CNFs with high aspect ratio extracted from poplar flour were used to reinforce PLA by extrusion. To solve the dispersion problem of CNFs in PLA matrix, the PLA water slurry was premixed with the CNFs followed by freeze drying prior to the extrusion. To improve the interfacial compatibility and impact toughness of the composite, Surlyn ionomer was added into the nanocomposites as compatibilizer during the compounding process. Surlyn ethylene ionomers used in this study are copolymers of ethylene and methacrylic/acrylic acid that has been partially neutralized by metal cations, such as zinc or sodium. ${ }^{10}$ Surlyn ionomers have extraordinary toughness and can improve the interfacial bonding strength in advanced composites. ${ }^{11-13}$ Recently, ionomers have been used as compatibilizers for nanocomposites based on PP, PET, PBT, and a variety of thermoplastics. ${ }^{14}$ As compared to the interfacial modification effect of ionomer, maleic anhydride grafted polyethylene (MAPE) was used as the traditional coupling agent for the CNFs/PLA composites. To investigate the reinforcing effect of the ultra-long CNFs, the poplar flour/PLA composite was also fabricated as control. The capillary rheological tests of the nanocomposites were performed to assess the influence of ionomer on the viscosity and flowability of the CNFs/PLA melts. Mechanical tests were carried out to evaluate the effectiveness 
in impact toughness and bending properties. Fracture surfaces of the nanocomposites were observed with FE-SEM to study the dispersion of CNFs and understand the failure behavior of the nanocomposite.

\section{Materials and methods}

\subsection{Materials}

The poplar flour with the size of 60-80 mesh was obtained from Nanjing Jufeng Advanced Materials Co., Ltd. (Nanjing, China). Polylactic acid (PLA), Nature Works TM 4032 D, was supplied by Nanjing JuFeng Advanced Materials Co., Ltd. (Nanjing, China). The glass transition temperature $\left(T_{\mathrm{g}}\right)$ and melting point $\left(T_{\mathrm{m}}\right)$ was $55^{\circ} \mathrm{C}$ and $180^{\circ} \mathrm{C}$, respectively. The melt flow index (MFI) was $6 \mathrm{~g}$ per $10 \mathrm{~min}\left(190{ }^{\circ} \mathrm{C}, 2.16 \mathrm{~kg}\right)$. MAPE with MFI of $6.4 \mathrm{~g}$ per $10 \mathrm{~min}$ and grafting ratio of $0.8 \%$ was purchased from Nanjing Deba Chemical Industry Company (Nanjing, China). Surlyn sodium ionomer (8940) with MFI of $2.8 \mathrm{~g}$ per $10 \mathrm{~min}$, density of $0.94 \mathrm{~g} \mathrm{~cm}^{-3}$, and melt point $\left(T_{\mathrm{m}}\right)$ of $78{ }^{\circ} \mathrm{C}$ was purchased from Du Pont China Holding Co., Ltd. (Shenzhen, China). All laboratory grade chemical reagents were purchased from Nanjing Chemical Reagent Co., Ltd. (Nanjing, China).

\subsection{Preparation of CNFs}

The preparation of poplar cellulose nanofiber was mainly divided into two steps based on our previously reported methods, including chemical pretreatment and grinding nanofibrillation. ${ }^{15}$ After chemical treatment, the water slurry with $1 \mathrm{wt} \%$ cellulose nanofibers was passed through a grinder (MKCA6-2, Masuko Sangyo Co., Japan) for 20 times with the grinding stone at $1800 \mathrm{rpm}$.

\subsection{Preparation of CNFs/PLA/ionomer composite}

Before the compounding process, different contents PLA powders (60-80 mesh) were added into the $1 \mathrm{wt} \%$ CNFs water slurry and continuously stirred by a magnetic stirrer at $75{ }^{\circ} \mathrm{C}$ for $1 \mathrm{~h}$. After the water preblending process, the slurry was dehydrated by vacuum filtration with a Buchner funnel and then freeze-dried. Subsequently, the nanofibers/PLA mixture was fully broken using a blender and fed into a HAKKE minilab (HAKKE minilab II, Thermo Fisher Scientific, Germany) for capillary rheological test. Surlyn ionomer/MAPE was added as coupling agent for each formulation. The extrusion temperature was set at $180{ }^{\circ} \mathrm{C}$. The rotating screw speed was changed from $10 \mathrm{rpm}$ to $100 \mathrm{rpm}$. The poplar flour/PLA/ionomer composite was also prepared as control. After the capillary rheological test, the composite samples were extruded through a rectangular die with the sectional dimension of $3.5 \mathrm{~mm} \times 1 \mathrm{~mm}$. The samples were crushed into powder by a blender and then hot-pressed at $170{ }^{\circ} \mathrm{C}$ for $5 \mathrm{~min}$ with a press vulcanizer. Finally, the samples were cooled to room temperature. The samples formulation is listed in Table 1 . The total weight of the composite was $6 \mathrm{~g}$ for each formulation.
Table 1 The formulation of prepared composites

\begin{tabular}{llll}
\hline & CNFs/poplar flour (\%) & PLA (\%) & Na ionomer/MAPE (\%) \\
\hline 1 & 10 & 86 & 4 \\
2 & 20 & 76 & 4 \\
3 & 30 & 66 & 4 \\
4 & 40 & 56 & 4 \\
5 & 30 & 70 & 0 \\
6 & 30 & 68 & 2 \\
7 & 30 & 64 & 6
\end{tabular}

\subsection{Characterization and testing}

2.4.1 CNFs. The morphologies of poplar flour and CNFs were observed using FE-SEM (HITACHI S-4860, HITACHI, Japan). Prior to FE-SEM observations, the samples were kept overnight in a vacuum oven at $30{ }^{\circ} \mathrm{C}$ and then coated with gold for $30-60 \mathrm{~s}$ to avoid charging. The acceleration voltage was $3 \mathrm{kV}$ and the coating current was $10 \mathrm{~mA}$.

Fourier transform infrared (FTIR) spectra were measured on a Nicolet iS10 FTIR spectrometer (Thermo Scientific Inc., America) equipped with a single reflection attenuated total reflectance (ATR) system. Poplar flour and CNFs were tested in the wave number range of $4000-500 \mathrm{~cm}^{-1}$ at a resolution of 2 $\mathrm{cm}^{-1}$.

2.4.2 Composites. The bending properties were tested according to the ASTM D 790-2010 standard using a universal materials testing machine (AG-10TA, Shimadzu, Japan) with the span of $40 \mathrm{~mm}$ and the crosshead speed of $1 \mathrm{~mm} \mathrm{~min}^{-1}$. The compression-molded samples were cut into strips with $50 \mathrm{~mm}$ long, $6 \mathrm{~mm}$ wide and $2 \mathrm{~mm}$ thick. At least 6 replications were tested for each measurement.

The impact toughness of the composites was measured in accordance with ASTM D 256-2010 with an izod impact test machine (QJBCX, Shanghai Qingji Instrumentation Technology Co., Ltd, China) at room temperature. At least 4 replications were tested for each measurement.

Pure PLA and the PLA/CNFs/ionomer nanocomposite were characterised by a Nicolet iS10 FTIR spectrometer (Thermo Scientific Inc., America) equipped with a single reflection ATR system. Scans were recorded in the range of $4000-500 \mathrm{~cm}^{-1}$ with a resolution of $2 \mathrm{~cm}^{-1}$ for each sample.

The fracture surface and surface of different composites were observed by FE-SEM. The nanocomposite samples were frozen in liquid nitrogen then quickly broken. Prior to FE-SEM observations, the samples were kept overnight in a vacuum oven at $30^{\circ} \mathrm{C}$ and then coated with gold for $30-60 \mathrm{~s}$ to avoid charging.

\section{Results and discussion}

\subsection{Characterization of cellulose nanofibers}

3.1.1 FE-SEM. Fig. 1 shows the FE-SEM images of the poplar flour and the extracted cellulose fibers after grinding treatment. The surface of the poplar flour is very rough with some pits (Fig. 1a). The nanofibers are well-individualized after the chemical and physical treatments. A classical web-like 

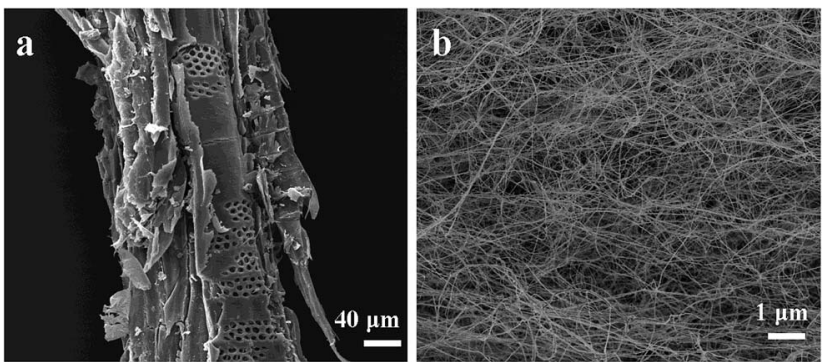

Fig. 1 FE-SEM images of (a) poplar flour and (b) poplar CNFs.

network structure was observed in all the nanofibers. Moreover, a very long entangled cellulosic filament was found. The diameter of the CNFs is between $30-100 \mathrm{~nm}$. The SEM observation also reveals that the length of most CNFs is a few microns. Hence the aspect ratio of poplar CNFs is up to 5002000. It indicates that nanofibers with high aspect ratio were successfully obtained from poplar flour.

3.1.2 FTIR. Fig. 2 represents the FTIR spectra of raw poplar flour and obtained cellulose nanofibers. The spectrum of the poplar flour is very different from that of the isolated CNFs. The dominant peak at $3406 \mathrm{~cm}^{-1}$ is attributed to the $-\mathrm{OH}$ stretching vibration, and the peak at $2890 \mathrm{~cm}^{-1}$ is ascribed to the $-\mathrm{CH}_{2}$ stretching vibration. The peaks at $1636 \mathrm{~cm}^{-1}$ and $1460 \mathrm{~cm}^{-1}$ represent the stretching vibration of $\mathrm{C}=\mathrm{O}$ and $\mathrm{O}-\mathrm{CH}_{3}$ of lignin, respectively. In addition, the absorption peaks at $1370 \mathrm{~cm}^{-1}$ and $1061 \mathrm{~cm}^{-1}$ originate from the flexural vibration of $-\mathrm{CH}$ and stretching vibration of $\mathrm{C}-\mathrm{O}$ of cellulose, respectively. Compared to the spectrum of poplar flour, the peak of CNFs at $2890 \mathrm{~cm}^{-1}$ disappears completely (Fig. 2b), indicating that all the hemicellulose have been sufficiently removed. However, the peak at $1636 \mathrm{~cm}^{-1}$ was observed due to some residual lignin in the nanofibers.

\subsection{Characterization of composites}

3.2.1 Capillary rheological properties. Rheology has been widely used to assess the morphology and interfacial status of polymer blends, but the effective rheological methods have not yet been explored to evaluate plant filler dispersion and interfacial status. ${ }^{16}$ In addition, additives are frequently used to modify processing or end properties of fiber/matrix composites. However, their effects have not been largely assessed from the rheological viewpoint. According to some reports, increasing the loading of nanofibers over $30 \mathrm{wt} \%$ is a simple method to improve the mechanical performance of polymer nanocomposites. ${ }^{17}$ Hence, the $30 \mathrm{wt} \%$ fiber content is a key factor in achieving the high performance of the CNFs/PLA composite. For $30 \mathrm{wt} \% \mathrm{CNFs} / \mathrm{PLA}$ composite with different levels of sodium ionomer, the viscosity dependence on the shear rate is presented in Fig. 3a. At lower shear rate $\left(<200 \mathrm{~s}^{-1}\right)$, an obvious decrease in viscosity with increasing shear rate can be observed, which indicates CNFs/PLA melts belong to pseudoplastic fluid of non-Newtonian fluid and reveal a "shear thinning" behavior. It can also be found that the viscosity of the nanocomposite decreases with the increase of sodium ionomer content, which demonstrates ionomer can improve the flowability of the CNFs/ PLA melts and reduce energy consumption for the productive process. Lower viscosity means better flowability and dispersion state. ${ }^{18}$ The result can be explained that ionomer in the CNFs/ PLA melt can reduce the entanglement of the nanofibers by the interactions between the carboxyl groups of sodium ionomer and hydroxyl groups of cellulose fibers. ${ }^{19}$

The effects of different additives on the rheological performance of $30 \mathrm{wt} \%$ CNFs/PLA composite are illustrated in Fig. $3 \mathrm{~b}$. The viscosity of composite with $4 \mathrm{wt} \%$ MAPE is slightly higher than the counterpart with $4 \mathrm{wt} \%$ sodium ionomer. MAPE plays a role in the interfacial compatibilization for the CNFs and PLA matrix, and CNFs coated by MAPE form a plastic lamina, reducing the viscosity of the composite melts. Compared to MAPE, sodium ionomer can reduce the viscosity and improve the flowability of the composite. The decrease in viscosity of the composite melts with ionomer can be attributed to the fact that ionomer facilitates the homogeneous dispersion of entangled CNFs in the PLA matrix as a lubricant. ${ }^{20}$ It is reported that elastomeric modifiers can improve the flow of fiber/polymer composites as lubricant at the polymer/fiber interface. ${ }^{21}$
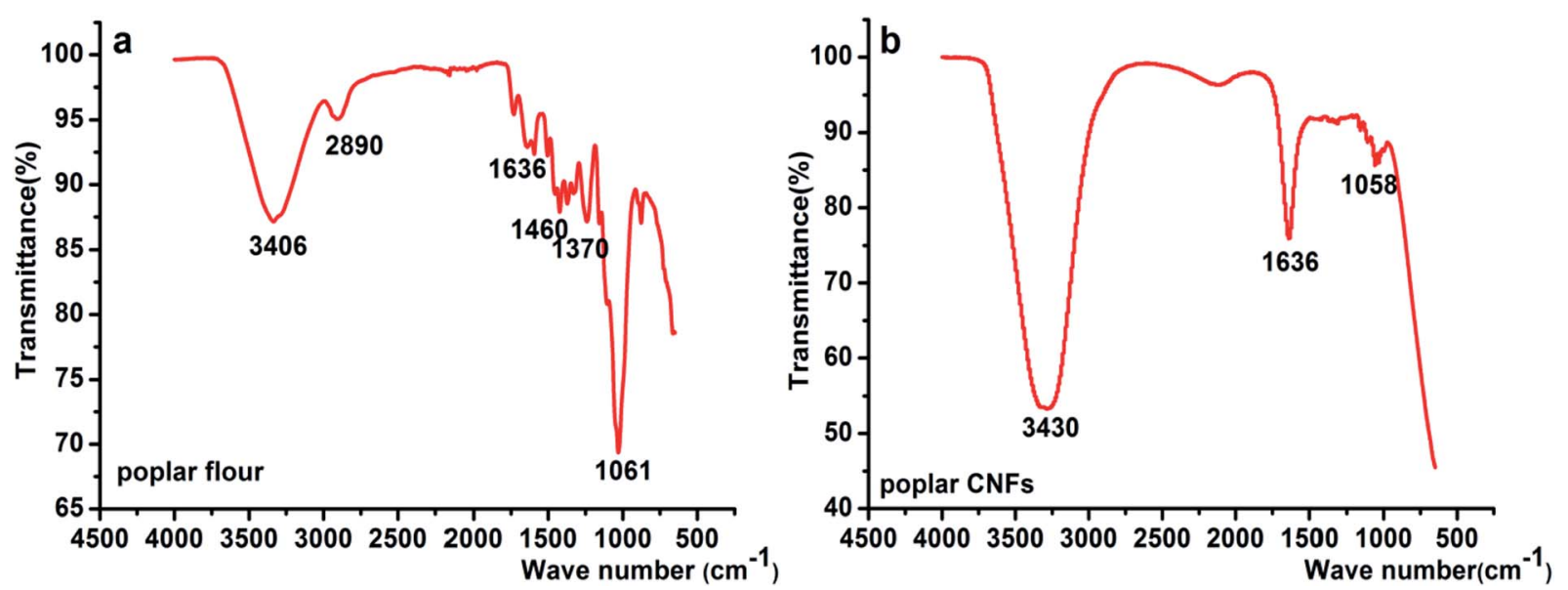

Fig. 2 FTIR spectra of (a) poplar flour and (b) poplar CNFs. 

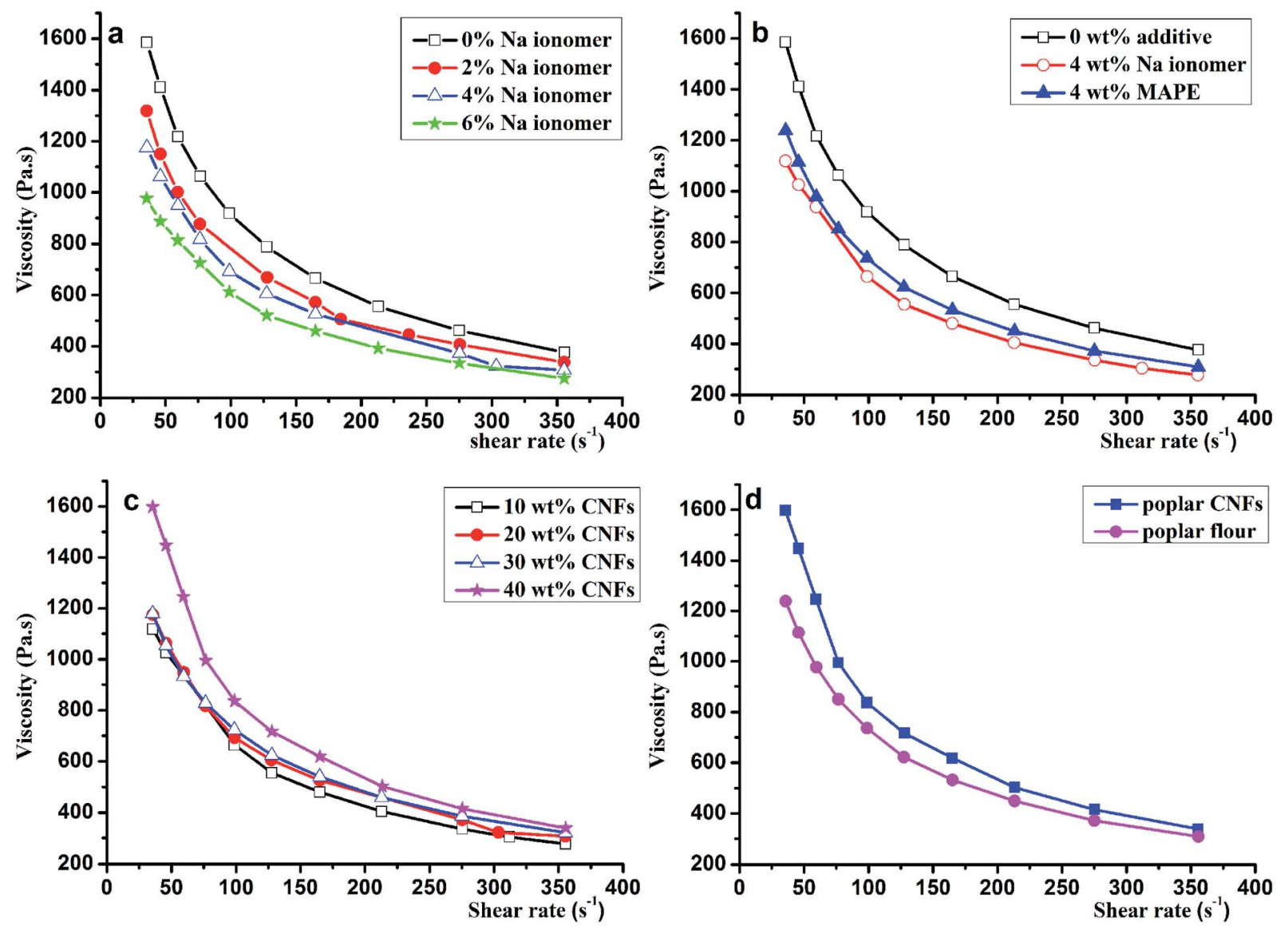

Fig. 3 The viscosity of (a) 30 wt\% CNFs/PLA composites with different Na ionomer contents, (b) 30 wt\% CNFs/PLA composites with different additives, (c) CNFs/PLA/4 wt\% Na ionomer composites with different CNFs contents, and (d) 30 wt\% CNFs/PLA/4 wt\% Na ionomer composite and 30 wt\% poplar flour/PLA/4 wt\% Na ionomer composite.

Viscosity of fiber/polymer composites depends on fiber loading and the nature of fibers. ${ }^{22}$ Fig. $3 \mathrm{c}$ shows the effect of CNFs loading on the viscosity of the nanocomposite with $4 \mathrm{wt} \%$ sodium ionomer. The viscosity of the nanocomposite rises with increasing the CNFs concentration. The presence of CNFs disturbs the normal flow of the composite melts and hinders the mobility of chain segments. Thus, relatively high CNFs concentration can worsen the dispersion of the entangled CNFs in the polymer melt, and increase the viscosity of the nanocomposites. In addition, it can be found that the pseudoplastic behavior is enhanced by increasing the CNFs content.

The effect of particle size on the viscosity is presented in Fig. 3d. It can be noticed that the viscosity of the CNFs composite is higher than that of the poplar flour composite when the formulation of the composite is fixed. Compared with the poplar flour, the aspect ratio of CNFs is high and there are more hydroxyl groups on the surface of molecular chains to form hydrogen bonding. The poplar flour is more easily aligned and distributed along the direction of flow than CNFs, thus the probability of fiber-fiber collisions is much less, leading to the large decrease in the viscosity of the nanocomposites. ${ }^{23}$

\subsubsection{Mechanical properties}

Flexural performance. In order to investigate the reinforcing effect of CNFs on PLA, the flexural properties of fabricated composites with different content of fillers and additives are studied. The bending strength (MOR) and bending modulus (MOE) of neat PLA and prepared composites are illustrated in Fig. 4. The effects of sodium ionomer/MAPE content on the MOR and MOE of the poplar CNFs/PLA composites at the loading of $30 \mathrm{wt} \%$ CNFs are shown in Fig. $4 \mathrm{a}$ and b. It can be seen that both the sodium ionomer and MAPE can improve MOR and MOE the CNFs/PLA composites. Because they are amphiphilic polymers and can act as coupling agents, the interfacial bonding between the PLA matrix and CNFs is enhanced. The esterification reaction of the carboxyl groups on the molecular side chain of sodium ionomer with hydroxy groups on the surface of poplar CNFs occurs through the electrostatic bonding between two polar groups. ${ }^{19}$ The non-polar molecular chains of sodium ionomer can be entangled with the molecular chains of PLA matrix due to the miscibility between ionomer and PLA. ${ }^{24}$ It can also be found that MOR and MOE of the composite with sodium ionomer are higher than those of the composite with MAPE. These results imply that Surlyn ionomer is a more effective coupling agent as compared to MAPE, leading to the high stress transfer efficiency from the matrix to the fiber. It can be inferred that the ionic bonding between the cations and acid groups on the polymer chains sets up a network structure, which enhances flexural strength of the 

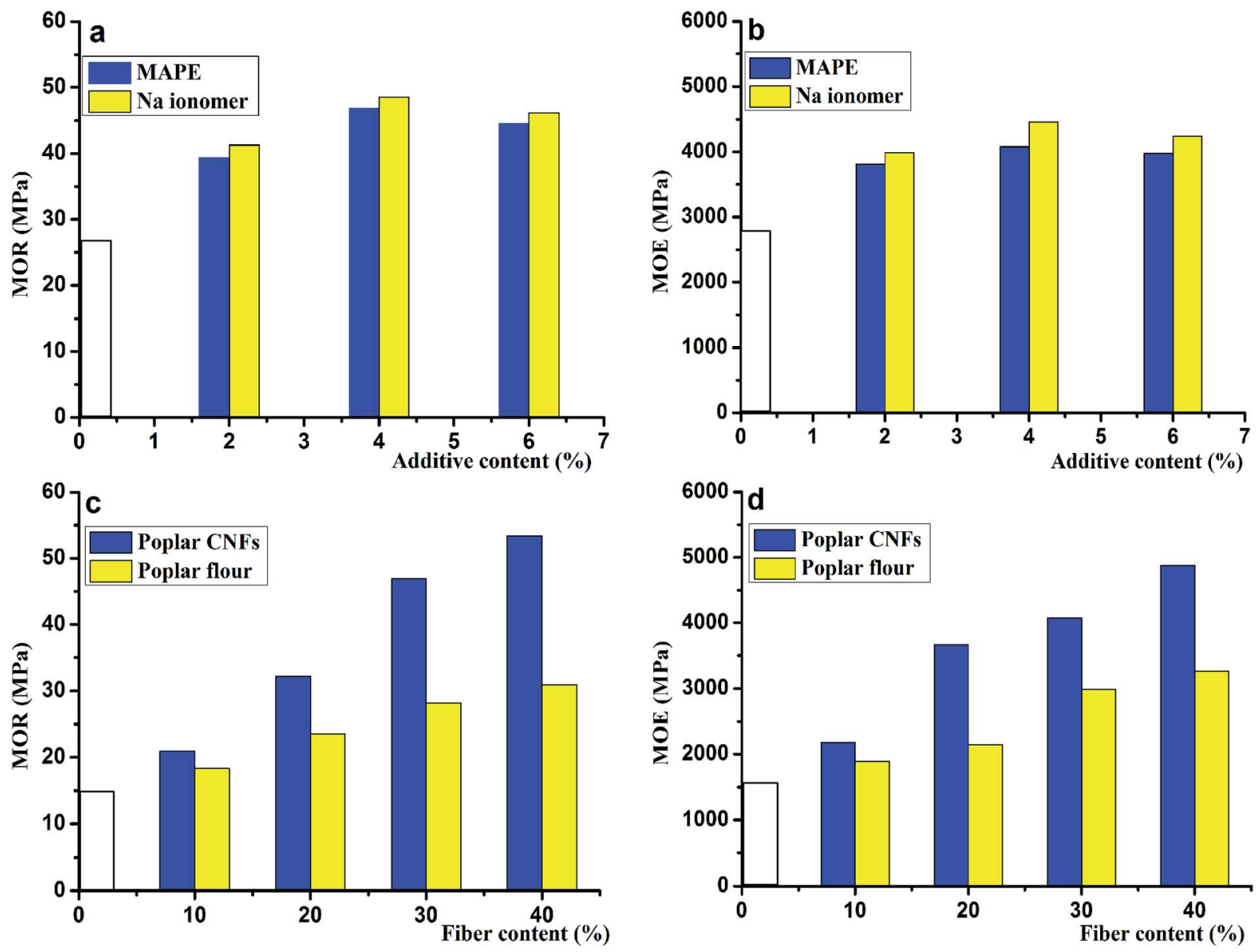

Fig. 4 MOR and MOE of ( $a$ and b) 30 wt\% CNFs/PLA/ionomer composites with different additive contents and (c and d) poplar CNFs/PLA/4 wt\% $\mathrm{Na}$ ionomer composite and poplar flour/PLA/4 wt\% Na ionomer composite with different fiber contents.

composites. ${ }^{25}$ Furthermore, MOR and MOE of the composite with $4 \mathrm{wt} \%$ ionomer are higher than those of the composite with $2 \mathrm{wt} \%$ ionomer, while a slight decrease in MOR and MOE of the composite with $6 \mathrm{wt} \%$ ionomer can be found. The decrease can be interpreted as that the incorporation of ionomers with low modulus can make the composites much more ductile. ${ }^{26} \mathrm{As}$ is well known, the modulus of polymers generally will decrease when polymers are toughened. Structure details, such as the molecular weight, ionic monomer content, and degree of neutralization of the ionomers, remain to be studied for future application of the ionomer techniques.

The effects of particle size and fiber content on the MOR and MOE of the composites with $4 \mathrm{wt} \%$ sodium ionomer are presented in Fig. $4 \mathrm{c}$ and d. It is seen that the MOR and MOE of the CNFs/PLA composite are much higher than those of poplar flour/PLA composite. It was reported that aspect ratio has a great effect on strength and stiffness of fiber/matrix composites. ${ }^{27}$ The length and aspect ratio of poplar CNFs are much higher than those of poplar flour, leading to much better mechanical properties. Compared to neat PLA, the MOR and MOE increase significantly with increasing content of poplar CNFs. When the CNFs content is up to $40 \mathrm{wt} \%$, the MOR and MOE of the poplar CNFs/PLA/4 wt\% Na ionomer composites are $53.4 \mathrm{MPa}$ and $4878 \mathrm{MPa}$, increasing by $260.8 \%$ and $212.3 \%$ as compared to neat PLA, respectively. The improvement of the mechanical performance of the nanocomposites is ascribed to the formation of a rigid fibers network, which maximizes stress transfer across interfaces to promote the mechanical strength. ${ }^{28}$ This result demonstrates that homogenous dispersion of CNFs can be achieved by using the water preblending method. Furthermore, the sodium ionomer acts as a bridge between the hydrophilic CNFs and hydrophobic PLA, improving eventually the interfacial strength of the composite.

Impact toughness. Fig. 5 represents the impact toughness of the prepared samples. The effect of sodium/MAPE content on the impact toughness of the $30 \mathrm{CNFs} / \mathrm{PLA}$ composites is shown in Fig. 5a. It can be seen that the impact toughness of the composite increases with increasing ionomer content. In addition, the impact toughness of the nanocomposites with sodium ionomer is extremely high as compared to the composites with MPAE. Ionomers are polymers containing a small amount of ionic groups, which are distributed along a nonionic hydrocarbon chain. ${ }^{29}$ High impact toughness is one of the most important physical properties of ionomer. ${ }^{25}$ It mainly depends on the presence of ionic aggregate. When the ionic aggregate concentration decreases, the chain mobility and energy dissipation will decrease, making the ionomer much more brittle. But at a relatively high ionic aggregate 

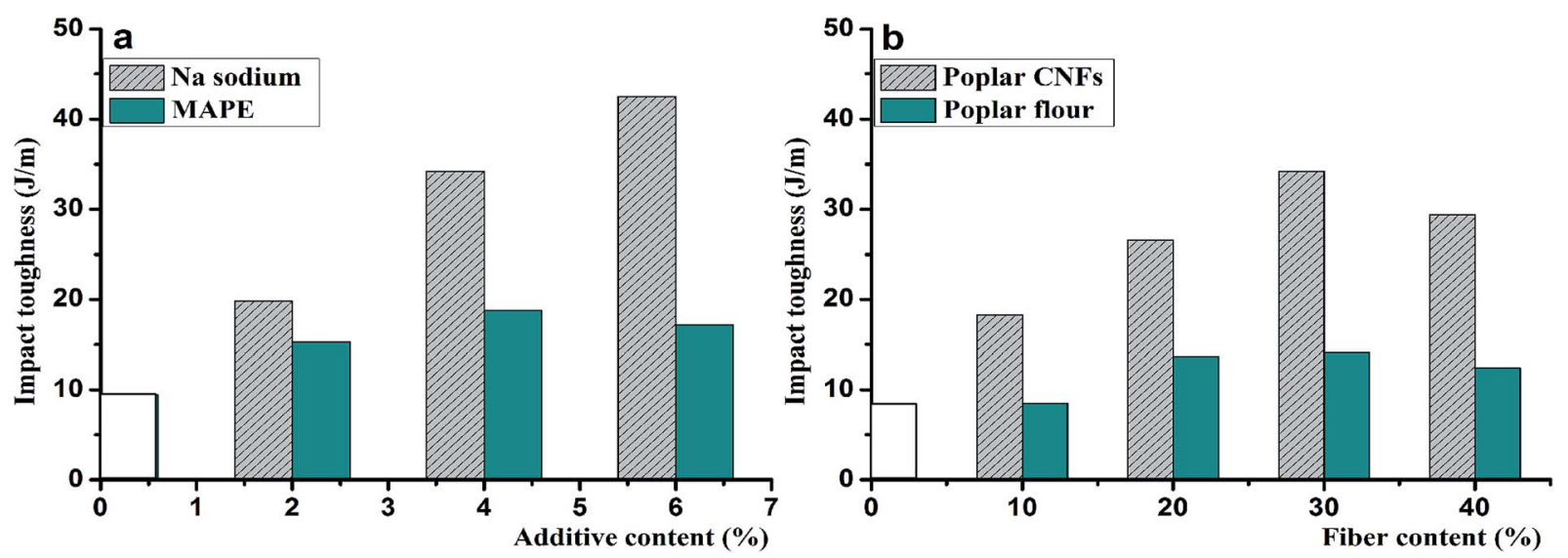

Fig. 5 Impact toughness of (a) 30 wt\% CNFs/PLA/ionomer composites with different additive contents and (b) poplar CNFs/PLA/4 wt\% Na ionomer composite and poplar flour/PLA/4 wt\% Na ionomer composite with different fiber contents.

concentration, large ionic clusters will play the dominant role in the system, and improve the impact strength of ionomer..$^{30}$ During the impact fracture process of the composite with ionomer, both crazing and shear yielding phenomena could be clearly observed. In addition, it was reported that ionomers can promote the nucleation of PLA matrix, leading to the improvement of the impact strength of the composite. ${ }^{24}$ Above all, the unique structure and morphology of the ionomers result in their serving as crosslink points to form networks of molecular chains, which can absorb the impact energy and prevent the crack initiation.

The effects of particle size and fiber content on the impact toughness of the composites with $4 \mathrm{wt} \%$ sodium ionomer are presented in Fig. 5b. It can be found that the impact toughness increases with increasing the content of poplar CNFs/flour below $30 \mathrm{wt} \%$. However, a decrease in impact toughness can be found when the fiber content is above $30 \mathrm{wt} \%$. The highest impact toughness of the $30 \mathrm{wt} \% \mathrm{CNFs} / \mathrm{PLA}$ composite is up to $34.2 \mathrm{~J} \mathrm{~m}^{-1}$, increasing by $302.3 \%$ as compared to neat PLA. If the nanofibers are uniformly dispersed in the matrix, the refined nanofibers network structure can absorb a large amount of energy in the process of fracture, leading to a remarkable improvement of impact strength of the nanocomposite. It also can be seen that the impact toughness of the CNFs/PLA composites is much higher than that of the poplar flour/PLA composites. The impact toughness of the composites is strongly dependent on aspect ratio and content of fiber. The short fibers in the matrix provide the points of stress concentrations, thus generating the sites for crack initiation and potential failure. ${ }^{31}$ For the CNFs/PLA composite, the impact toughness decreases above fiber content of $30 \mathrm{wt} \%$ due to the tendency of the agglomeration into larger aggregates at high CNFs content.

3.2.3 FTIR. The FTIR spectra of PLA and PLA/CNFs/ ionomer composite are shown in Fig. 6. For the pure PLA (Fig. 6a), the peaks at $2998 \mathrm{~cm}^{-1}, 2947 \mathrm{~cm}^{-1}$, and $1360 \mathrm{~cm}^{-1}$ are attributed to the stretching and bending vibrations of $-\mathrm{CH}_{2}$. The peak at $1746 \mathrm{~cm}^{-1}$ is ascribed to the $\mathrm{C}=\mathrm{O}$ stretching vibration. The peak at $1452 \mathrm{~cm}^{-1}$ corresponds to the $-\mathrm{CH}_{3}$ bending vibration. The peaks at $1182 \mathrm{~cm}^{-1}, 1120 \mathrm{~cm}^{-1}$, and $1040 \mathrm{~cm}^{-1}$ represent the $\mathrm{C}-\mathrm{O}-\mathrm{C}$ stretching vibrations. The peak at $870 \mathrm{~cm}^{-1}$ originates from the stretching vibration of the $\mathrm{C}-\mathrm{C}$ single bond. For the PLA/CNFs/ionomer composite (Fig. 6b), a broad absorption peak that is related to the hydroxyl group
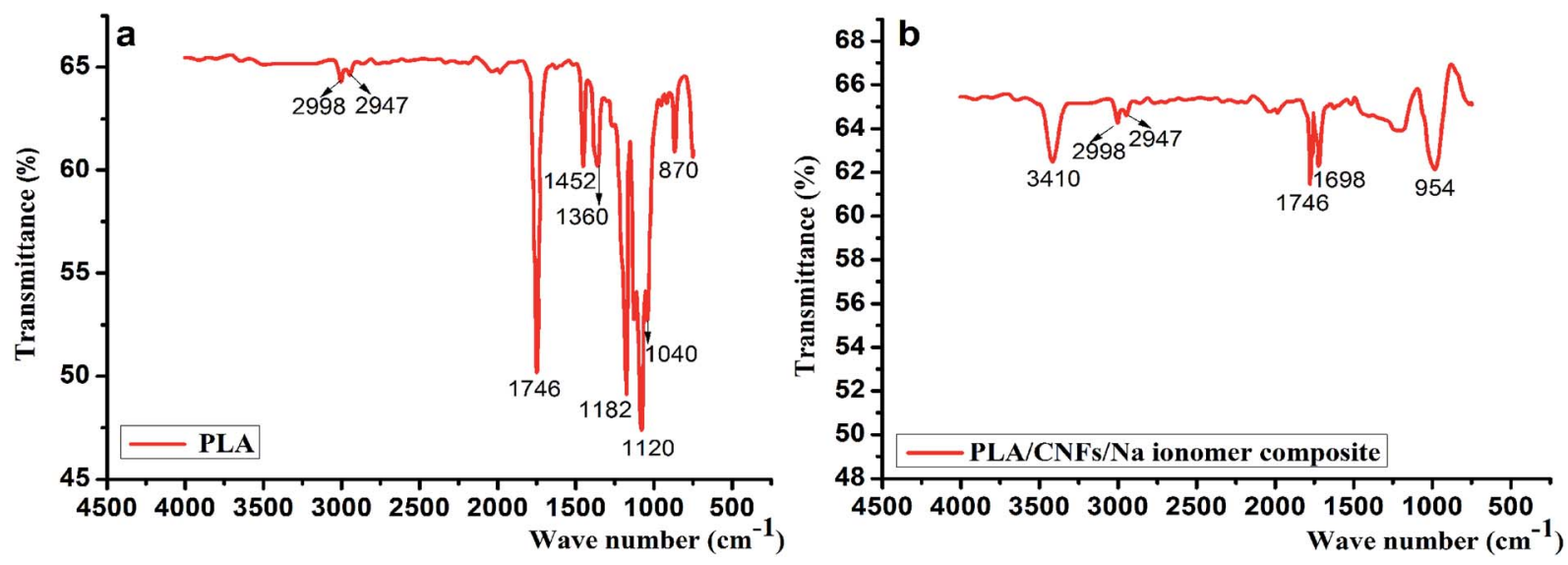

Fig. 6 FTIR spectra of (a) PLA and (b) PLA/CNFs/Na ionomer composite. 
was observed at $3410 \mathrm{~cm}^{-1}$. Compared to the FTIR spectrum of PLA, there is a new peak at $1698 \mathrm{~cm}^{-1}$ for the PLA/CNFs/ ionomer composite, suggesting the formation of $\mathrm{C}=\mathrm{O}$ linkage between CNFs and sodium ionomer. The characteristic absorption peak demonstrates that the carboxyl group on the molecular side chain of sodium ionomer esterified with hydroxy group of the surface of CNFs. In addition, the peaks at 1452 $\mathrm{cm}^{-1}, 1360 \mathrm{~cm}^{-1}, 1182 \mathrm{~cm}^{-1}, 1120 \mathrm{~cm}^{-1}$, and $1040 \mathrm{~cm}^{-1}$ almost disappear for the PLA/CNFs/ionomer composite.

3.2.4 FE-SEM. Fig. 7 shows FE-SEM images of the prepared composites. The fracture surface and surface of the $30 \mathrm{wt} \%$
CNFs/PLA/4 wt\% sodium ionomer composite are illustrated in Fig. 7a and b. It is observed that lots of ultra-long nanofibers are distributed uniformly and compactly on the fracture surface of the composite in the form of "spider web". Homogenous dispersion of CNFs with high aspect ratio is achieved by using the water preblending method. From the SEM images, the network structure of CNFs is not destroyed during the preblending and compounding processes. The surface morphology of the composite shows that poplar CNFs are mixed very well with the PLA matrix. By the water pretreatment method, hydrophilic nanofiber and hydrophobic PLA fibers can be
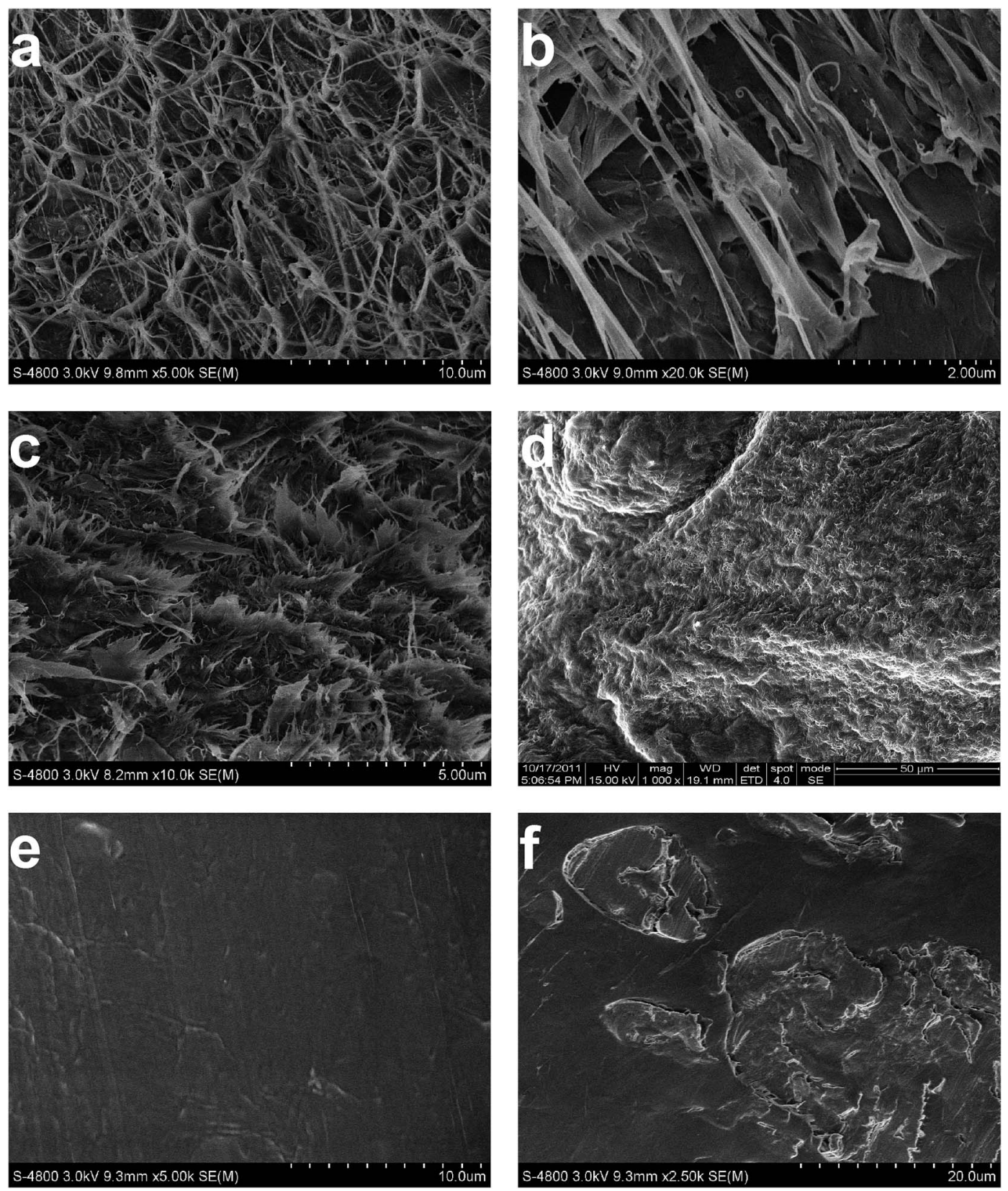

Fig. 7 FE-SEM images of (a) fracture surface of 30 wt\% CNFs/PLA/4 wt\% Na ionomer composite, (b) surface of 30 wt\% CNFs/PLA/4 wt\% Na ionomer composite, (c) fracture surface of $30 \mathrm{wt} \%$ poplar four/PLA/4 wt\% Na ionomer composite, (d) surface of $30 \mathrm{wt} \%$ poplar four/PLA/4 wt\% $\mathrm{Na}$ ionomer composite, (e) fracture surface of $30 \mathrm{wt} \%$ poplar four/PLA/4 wt\% MAPE composite, and (f) fracture surface of $30 \mathrm{wt} \%$ poplar four/ PLA/0 wt\% additive composite. 
uniformly dispersed in aqueous suspension. ${ }^{32}$ During the preblending process, nanofibers are adsorbed on PLA fibers, and entangled to form a network. Therefore, the electrostatic repulsion and steric hindrance between nanofibers and PLA matrix result in the homogenous dispersion of cellulose nanofibers. ${ }^{33}$

To study the toughening effect of ionomers, the fracture surface and surface morphologies of the $30 \mathrm{wt} \%$ poplar flour/ PLA $/ 4$ wt\% sodium ionomer composite are presented in Fig. $7 c$ and $d$. There are a great number of little fibrils on the fracture surface of the composite, showing the remaining traces of cold drawing during the impact failure (Fig. 7c). The rough fracture surface demonstrates the ductile fracture of the composites with ionomer. From Fig. 7d, poplar fibers are completely coated by polymers. There are hardly gap and void on the surface of the composite. During the period from deformation to fatal failure, large strain energy is uniformly dissipated in a wide area due to the adhesive strength between fibers and PLA matrix. Compared to the morphology of the CNFs/PLA/ionomer composite, a significant decrease in the length of fibers can be observed. For the CNFs/PLA/ionomer composite, the synergistic effect of the high-aspect-ratio CNFs with ionomer contributes to the excellent impact toughness of the composite.

Fig. 7e and f show the fracture surfaces of $30 \mathrm{wt} \%$ poplar flour/PLA/4 wt $\%$ MAPE composite and $30 \mathrm{wt} \%$ poplar flour/PLA/ 0 wt $\%$ additive composite, respectively. It can be seen the fracture surface of the composite with MAPE is very smooth, indicating the brittle crack propagation during the impact failure (Fig. 7e). There is no obvious void or hole around the interface region, which is attributed to the strong interaction between the PLA matrix and poplar fibers in the presence of MAPE as a compatibilizer. In contrast, the fracture surface of the composite without any additive is remarkably different. Fig. $7 f$ shows that there are lots of gaps and holes between fibers and matrix. It indicates that there are poor dispersion and weak interfacial bonding between the PLA matrix and poplar fibers.

\section{Conclusions}

The poplar CNFs/PLA/ionomer composite was prepared by extrusion. The rheological results showed the viscosity of the nanocomposite decreased with increasing sodium ionomer content, and the viscosity of the composite with ionomer was lower than that of the composite with MAPE. It demonstrated ionomer could improve significantly the flowability of the CNFs/ PLA melts due to the lubrication of ionomer.

The mechanical tests showed that the MOR and MOE of the composite with sodium ionomer were much higher than those of composite with MAPE. The highest MOR and MOE of the CNFs/PLA/ionomer composites were 53.4 MPa and $4878 \mathrm{MPa}$, increasing by $260.8 \%$ and $212.3 \%$ as compared to neat PLA, respectively. Moreover, the extremely high impact toughness of the CNFs/PLA/ionomer composite was achieved. The highest impact toughness of the CNFs/PLA/ionomer composite was up to $34.2 \mathrm{~J} \mathrm{~m}^{-1}$, increasing by $302.3 \%$ in comparison to neat PLA.
FE-SEM images showed homogenous dispersion of CNFs in PLA with the water preblending method. The rough fracture surface of the composite demonstrated the plastic deformation during impact fracture. The synergistic effect of the CNFs with high aspect ratio and ionomer contributed to the excellent impact toughness of the composite. In addition, there was no obvious gap/void on the interface of fibers and PLA matrix with the addition of ionomer. All these results indicated Surlyn ionomer can be used as an ideal compatibilizer, improving simultaneously both the flowability and the mechanical properties of cellulose nanofibers/PLA composite.

\section{Acknowledgements}

This work is financially supported by PhD Start-up Fund of Northwest A\&F University (Z109021613).

\section{References}

1 M. Jonoobi, J. Harun, A. P. Mathew, et al., Mechanical properties of cellulose nanofiber (CNF) reinforced polylactic acid (PLA) prepared by twin screw extrusion, Compos. Sci. Technol., 2010, 70, 1742-1747.

2 A. N. Nakagaito, A. Fujimura, T. Sakai, et al., Production of microfibrillated cellulose (MFC) reinforced polylactic acid (PLA) nanocomposites from sheets obtained by a papermaking-like process, Compos. Sci. Technol., 2009, 69, 1293-1297.

3 K. Azouz, B. E. Ramires, C. W. V. Fonteyne, et al., Simple method for the melt extrusion of a cellulose nanocrystal reinforced hydrophobic polymer, ACS Macro Lett., 2012, 1, 236-240.

4 P. Dhar, D. Tarafder, A. Kumar, et al., Thermally recyclable polylactic acid/cellulose nanocrystal films through reactive extrusion process, Polymer, 2016, 87, 268-282.

5 A. N. Frone, S. Berlioz, J. F. Chailan, et al., Morphology and thermal properties of PLA-cellulose nanofibers composites, Carbohydr. Polym., 2013, 91, 377-384.

6 N. Herrera, A. P. Mathew and K. Oksman, Plasticized polylactic acid/cellulose nanocomposites prepared using melt-extrusion and liquid feeding: mechanical, thermal and opticalproperties, Compos. Sci. Technol., 2015, 106, 149-155.

7 M. Kowalczyk, E. Piorkowska, P. Kulpinski, et al., Mechanical and thermal properties of PLA composites with cellulose nanofibers and standard size fibers, Composites, Part A, 2011, 42, 1509-1514.

8 P. Tingaut, T. Zimmermann and F. Lopez-Suevos, Synthesis and characterization of bionanocomposites with tunable properties from poly(lactic acid) and acetylated microfibrillated cellulose, Biomacromolecules, 2010, 11, 454-464.

9 L. R. Tang, B. Huang, N. T. Yang, et al., Organic solvent-free and efficient manufacture of functionalized cellulose nanocrystals via one-pot tandem reactions, Green Chem., 2013, 15, 2369-2373. 
10 B. A. Morris and J. C. Chen, The stiffness of ionomers: How it is achieved and its importance to flexible packaging applications, SPE Antec., 2003, 3157-3161.

11 H. Akimoto, T. Kanazawa, M. Yamada, et al., Impact fracture behavior of ethylene ionomer and structural change after stretching, J. Appl. Polym. Sci., 2001, 81, 1712-1720.

12 E. R. Sadiku and R. D. Sanderson, Variables that determine the fiber-matrix bond strength in ethylene-type ionomer composites, Macromol. Mater. Eng., 2001, 286, 472-479.

13 S. Matsuda, M. Hojo, S. Ochiai, et al., Effect of ionomer thickness on mode I interlaminar fracture toughness for ionomer toughened CFRP, Composites, Part A, 1999, 30A, 1311-1319.

14 G. D. Barber, B. H. Calhoun and R. B. Moore, Poly(ethylene terephthalate) ionomer based clay nanocomposites produced via melt extrusion, Polymer, 2005, 17, 6706-6714.

15 J. J. Li, D. G. Li, Z. Q. Song, et al., Preparation and properties of wood plastic composite reinforced by ultralong cellulose nanofibers, Polym. Compos., 2016, 37, 1206-1215.

16 T. Q. Li and M. P. Wolcott, Rheology of HDPE-wood composites. I. Steady state shear and extensional flow, Composites, Part A, 2004, 35, 303-311.

17 K. Y. Lee, Y. Aitomaki, L. A. Berglund, et al., On the use of nanocellulose as reinforcement in polymer matrix composites, Compos. Sci. Technol., 2014, 105, 15-27.

18 J. J. Li, Z. Q. Song, D. G. Li, et al., Cotton cellulose nanofiberreinforced high density polyethylenecomposites prepared with two different pretreatment methods, Ind. Crops Prod., 2014, 59, 318-328.

19 J. K. Pandey, C. S. Kim, W. S. Chu, et al., Preparation and structural evaluation of nano reinforced composites from cellulose whiskers of grass and biodegradable polymer matrix, J. Compos. Mater., 2012, 46, 653-663.

20 H. Hosseinkhanli, J. Aalaie, M. Abdollahi, et al., Thermal, mechanical, and barrier properties of polyethylene/Surlyn/ oganoclay nanocomposites blown films prepared by different mixing methods, J. Vinyl Addit. Technol., 2015, 21, 60-69.

21 S. Dong, S. Sapieha and H. P. Schreiber, Rheological properties of corona modified cellulose/polyethylene composites, Polymer Engineering and Science, 1992, 32, 1734-1739.
22 M. Sain, V. Khunova, J. Hurst, et al., The influence of fillers on composite rheological properties, Plasty Kauc., 1998, 35, 199-202.

23 O. Carneiro and S. J. M. Maia, Rheological behavior of (short) carbon fiber/thermoplastic composites. Part 1: The influence of fiber type, processing conditions and level of incorporation, Polym. Compos., 2000, 21, 960-969.

24 Y. L. Ma, G. P. Yang and L. S. Xie, Morphology, nonisothermal crystallization behavior and mechanical properties of polypropylene modified by ionomers, $J$. Macromol. Sci., Part B: Phys., 2014, 53, 1829-1845.

25 H. Akimoto, T. Kanazawa, M. Yamada, et al., Impact fracture behavior of ethylene ionomer and structural change after stretching, J. Appl. Polym. Sci., 2001, 81, 712-720.

26 T. Q. Li and N. Yan, Mechanical properties of wood flour/ HDPE/ionomer composites, Composites, Part A, 2007, 38, 1-12.

27 N. M. Stark and E. Rowlands, Effects of wood fiber characteristics on mechanical properties of wood/ polypropylene composites, Wood Fiber Sci., 2003, 35, 167174.

28 D. S. Li, Z. Y. Liu, M. AlHaik, et al., Magnetic alignment of cellulose nanowhiskers in an all-cellulose composite, Polym. Bull., 2010, 65, 635-642.

29 M. Bellinger, J. A. Sauer and M. Hara, Tensile fracture properties of sulfonated polystyrene ionomers. 1. Effect of ion content, Macromolecules, 1994, 27(6), 1407-1412.

30 E. R. Sadiku and R. D. Sanderson, Variables that determine the fiber-matrix bond strength in ethylene-type ionomer composites, Macromol. Mater. Eng., 2001, 286, 472-479.

31 J. J. Li, Y. Q. Gao, J. F. Zhao, et al., Homogeneous dispersion of chitin nanofibers in polylactic acid with different pretreatment methods, Cellulose, 2017, 24, 1705-1715.

32 A. N. Nakagaito and H. Yano, The effect of fiber content on the mechanical and thermal expansion properties of biocomposites based on microfibrillated cellulose, Cellulose, 2008, 15, 555-559.

33 S. Q. Wang, C. Wei, Y. Y. Gong, et al., Cellulose nanofiberassisted dispersion of cellulose nanocrystals @ polyaniline in water and its conductive films, RSC Adv., 2016, 6, 10168-10174. 